\title{
Properties of the Short Term Memory Structure
}

\author{
by \\ Eugen Tarnow, Ph.D. \\ 18-11 Radburn Road \\ Fair Lawn, NJ 07410 \\ etarnow@avabiz.com (e-mail)
}

\begin{abstract}
:
Properties of a short term memory structure are discovered in the data of Rubin, Hinton and Wenzel (1999): Cued-recall probability and search time are linearly related from 6 seconds to 666 seconds after stimulus presentation with a zero probability of cued-recall at 2.6 seconds and a $100 \%$ recall probability at 1.3 seconds. This linear relationship defines a short term memory structure which is a moving structure: the memory structure travels away from the starting point of the search (suggesting that the starting points of the search and storage are the same), decaying with a rate proportional to the time it takes to find the structure. The travel speed is slower than Brownian motion. The incorrect recall time saturates, giving an upper limit for the number of neurons involved in the short term memory structure of $3^{\star} 10^{8}$ using an interneuron transfer time or 4 msecs or $3^{\star} 10^{6}$ using an neuron-neuron transfer time of $20 \mathrm{msecs}$.
\end{abstract}




\section{Introduction}

Memory is often conceptually divided into different structures. For example, short term structures has information that lasts longer than what is captured on the retina but not as long as the information about our personal identity [1]. After many decades of work, some properties of short term memory structures are starting to emerge. The number of chunks that can be remembered is 4-6 (for a review, see [2]), the search time for chunks is proportional to the number of chunks, and the search is serial and exhaustive (for search properties of integer recognition, see [3]).

It should be possible to characterize the different memory structure by different time constants but these have been elusive. In 1996 that an effort was made to fit the various retention data in the literature with different types of curves just to see which was correct [4]. The authors realized that existing experimental data did not cover large enough differences in time and at the same time keep statistical noise low to show a preference for an algebraic decay, an exponential decay, or even an asymptotic constant. The authors took it upon themselves to come up with a set of experimental data over two orders of magnitude in time with small enough statistical error bars to be theoretically useful [5] (RHW). This invaluable source of information for memory researchers contains cued-recall and recognition probabilities and reaction times.

In this paper we will show that the RHW data tells us that the short term memory structure for cued-recall is a moving structure which decays at the same rate it takes to search and find the memory structure and we will extricate other properties of the short term memory structure as well.

\section{Properties of the short term structure in the RHW data}

\section{i) Reaction time is linearly related to probability of cued recall}

The original interest of the RHW authors was to settle once and for all how memory decays with time. They claimed to have settled the time dependence of cued-recall but not the time dependence of the corresponding reaction time. I decided to look at the data differently and understand how reaction time correlates with the probability of cued recall. Let us begin by considering the "correct answer" set in Tables A1 and A4 of [5]. I find an astonishingly good fit: the probability of cued recall is linearly related to the reaction time with $\mathrm{R}$ squared being $98 \%$ over two orders of magnitudes, 6 to 600 seconds (see Figure 1)! A recent item requires a total reaction time of about 1.3 seconds while an item that is typically no longer to be found for most participants requires 2.6 seconds (reaction time was defined by the latency until the first key was pressed to spell the recalled word). 


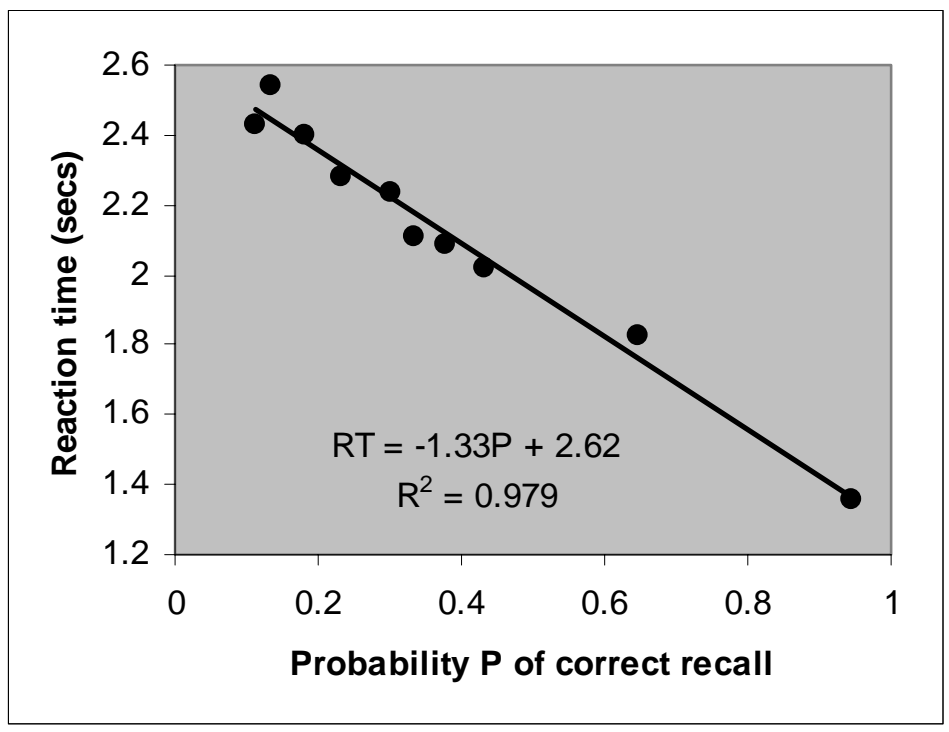

Figure 1: Reaction time as a function of the probability of correct recall.

\section{ii) The linear relationship defines a short term memory structure}

The linear relationship suggests that all of the data covered is somehow processing in the same way by a yet unknown mechanism for cued recall between 6 and 660 seconds. I am going to let this remarkable linear relationship serve as the definition of the short term memory structure, for now without knowing what it means.

\section{iii) The short term memory structure is a moving structure}

The linear relationship between search time and probability of correct cued recall tells us something about the geometry of the short term memory structure. Let us consider three possibilities. (For the rest of this paper, I will assume that the search speed is relatively constant. This does not seem improbable since the search speed could be relatively independent across the particular brain area which corresponds to the short term memory).

Possibility 1. A non-redundant randomly decaying memory structure fixed in space has a search time for correctly identified items which is independent of the probability of finding the item. The items are either there or not and if they are, they are in the same spot whether the probability of finding them is high or low and take the same time to find. This is clearly not the case for the memory structure in the data of RHW.

Possibility 2. A multiple redundant randomly decaying memory structure fixed in space would not show a linear relationship with the reaction time but rather the reaction time $R T \sim 1 / \mathrm{P}$ where $\mathrm{P}$ is the probability of cued-recall: for example, if there are two copies of an item randomly positioned, 
it would on the average take half the time to find the item as compared to if there were only one item and so forth: the probability of a correct answer is halved, the time to search for it should be doubled leading to $\mathrm{RT} \sim 1 / \mathrm{P}$.

Possibility 3 - the Moving Memory Model. A non-redundant randomly decaying memory structure moving away from the search starting point or a non-redundant stationary memory structure which decays in such a way that it is effectively moving, with an equal loss probability per distance traveled would give a linear relationship with reaction time: since a stationary decaying memory structure has a constant search time a moving memory structure has a linear relationship with reaction time.

iv) The storage and search processes: the corresponding starting points seem to be the same

In a cued-recall experiment there are two time scales of interest for this paper: the storage time (the time after the stimulus has been presented) and the search time needed to find the recalled item. Our finding is that the time it takes to find the structured memory increases substantially with the storage time suggests but does not prove that both search and storage starts in the same geographical place. It is consistent with a short term storage starting out at the starting point of the search process and slowly moving out from that point in a spherical fashion.

\section{v) The speed of the movement of the short term memory structure}

Since we assume that the search speed is relatively constant, we have to conclude that the movement of the decaying memory structure is clearly not constant (see Fig. 2). Rather it moves quickly at first and then slows down. Random motion of the nerve impulse would suggest a resulting movement proportional to $(t / T)^{0.5}$ where $T$ is the time between collisions (Brownian motion), in this case the time between neuron firings. In the RHW data, the movement can be described as a $(t / T)^{0.0667}$ dependence where $T$ is about $0.5 \mathrm{msec}$. That the speed is slower suggests that firings further away are much less likely rather than closer to the starting point, presumably because the further away from the starting point, the fewer neurons are firing and, correspondingly, the much fewer neurons can be made to fire. That $T$ is about $0.5 \mathrm{msec}$ is not inconsistent with possible interneuron signal times of $0.5 \mathrm{msec}$ to $0.1 \mathrm{sec}$ [6] though $4 \mathrm{msec}$ seems to be an acceptable minimum rate used for modeling purposes [7]. 


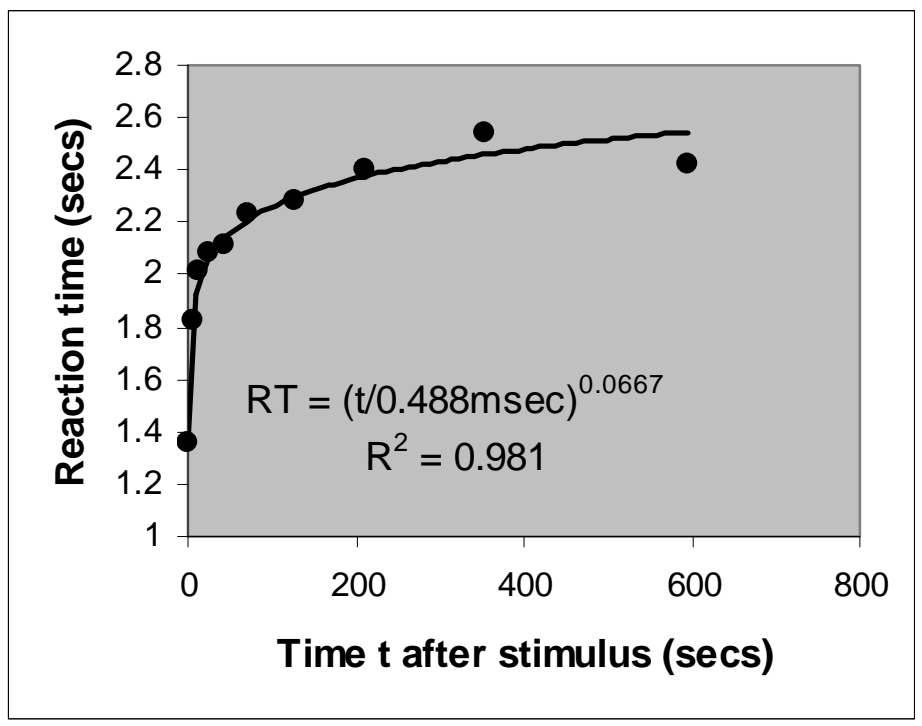

Figure 2. Movement of the decaying memory structure. The curve represents a two parameter power fit, moving $t=0.0$ to $t=0.05$ to avoid a divergence: Reaction time $R T=(t / 0.488 \mathrm{msec})^{0.0667}$.

\section{vi) The size of the short term memory structure}

Let us now apply the Moving Memory Model to the relationship between response time and "incorrect" cued-recall (See RHW, Table A5 - the data is shown in Fibure 3). As the reader can see (Figure 4), the response time is always larger than for correct recall, contradicting the earlier observation of exhaustive search [3]. When the recall probability is large it changes linearly just like the "correct" recall but when the recall probability decreases it becomes constant. What might be going on?

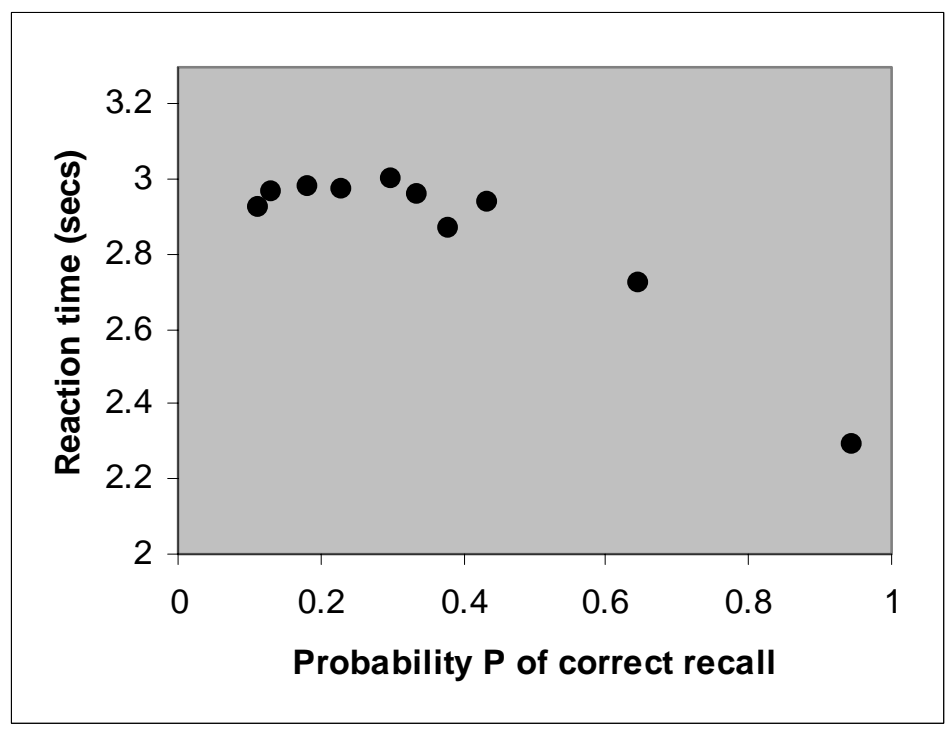


Figure 3: Reaction time as a function of the probability of incorrect recall.

I conclude that the "correct response" search is not exhaustive and that when no answer is found the search takes longer because it now is exhaustive. When the probability is high it goes beyond the correct search by about 0.9 seconds (the difference in RT between the incorrect and correct searches is shown in Figure 4 below). Presumably, the 0.9 seconds is the average extra time the RHW subjects used for the exhaustive search, it may have no other meaning than that.

That the incorrect search reaches a maximum may have a very important meaning: As the short term memory structure moves away from the starting point, it may get closer to some sort of a barrier - the end of a brain structure, for example. If this is the case, the exhaustive search would keep searching beyond the original moving location for, on the average 0.9 seconds, but only until it hits the end of the brain structure. It then has to stop.

We can estimate the time it takes to get to the end of the brain structure to be 3 seconds (from Figure 3 ) minus the reaction time at $\mathrm{P}=1$ (from Figure 1) or about 1.7 seconds. With a neuronal travel time of about 1 neuron length per $4 \mathrm{msec}$ [7] (20 msec [Chinese guy]), the number of neurons in the brain structure, assuming a spherical distribution for simplicity, can be estimated to be $4 / 3 \mathrm{PI}(1.7 / 0.004)^{\star \star 3}=3^{\star} 10^{8}\left(3^{\star} 10^{6}\right)$. This is not inconsistent with the current estimate of the total number of braincells of $10^{11}-10^{12}$.

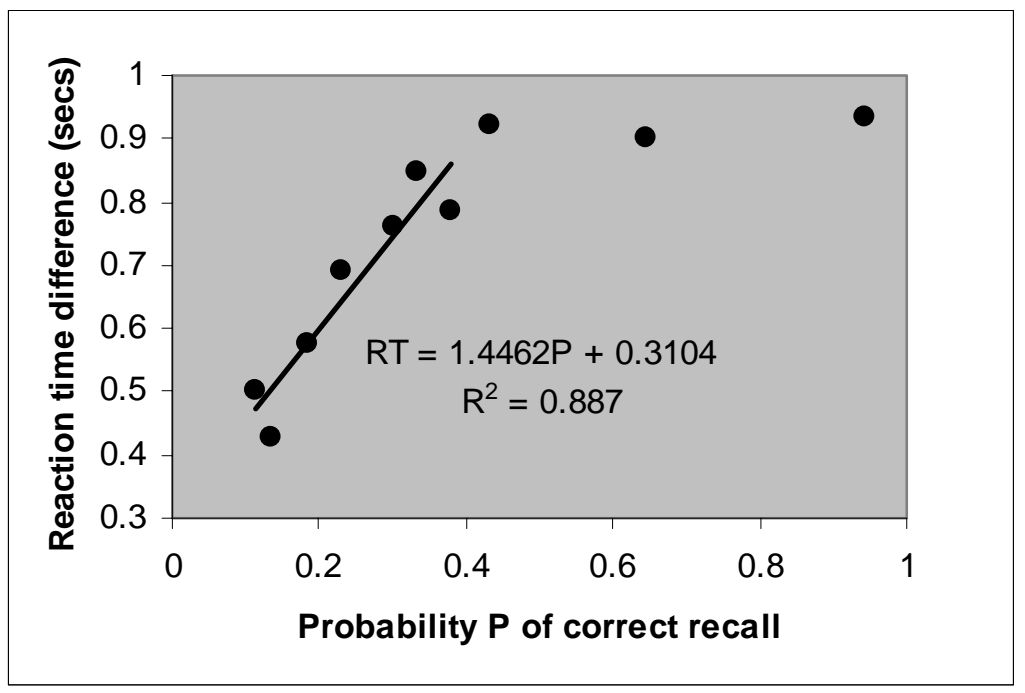

Figure 4: Difference in reaction time between incorrect and correct recall as a function of the probability of recall. 


\section{vii) Longest storage time for the short term memory structure}

The remarkable linear relationship of reaction time with probability of cued-recall gives us a limit on the time that something can be remembered in the short term memory structure before it is totally gone: From the fit in Figure 2, at $\mathrm{P}=0, \mathrm{RT}$ is 2.62 and the corresponding time is 912 seconds. Another way to affix a time to memory decay is to look at the time for the probability of recall to drop by $50 \%$ - it is about 16 seconds.

In the RHW paper [5], the authors concluded that the best fit to their recall-probability vs. time after stimulus presentation $w$ as an exponential fit with three different time scales associated with it, the smallest of which they felt corresponded to working memory $(T=1.15$ lags which is 6.9 seconds). I tend to think that the RHW authors made a mistake constructing a fit of 5 parameters to only 9 data points (they excluded the first data point) and that their experimental data, with its error bars, cannot distinguish between an algebraic decay (in which case there is no parameter with time units present) and exponential decay (where the "half-life" is given in terms of time) when one uses 5 parameters. To really prove their case for working memory at 6.9 seconds, they should include more experimental points around 6 seconds where the data undergoes large changes.

\section{Discussion}

There are other theories of memory that should be considered. Nelson Cowan pointed out a theory by Ratcliff [8], in which it takes a certain number of features to reach a criterion for detecting the item in memory. This might show a relationship of reaction time and probability of recall similar to the one in the RHW data but it is somewhat unlikely - one would not expect that a feature comparison would take that much time that it would slow down the reaction time by up to 1.3 seconds if the Sternberg data of integer comparison [3] shows that comparing integers take about 0.02 seconds per integer (which presumably would have several features as well).

\section{ACKNOWLEDGEMENT}

Thanks to Nelson Cowan and Stephen Schmidt for comments on the manuscript. 


\section{REFERENCES}

1. Neath, I (1998). "Human Memory". Brooks/Cole. Pacific Grove. P. 54.

2. Cowan, $N$ (2000). "The magical number 4 in short-term memory: A reconsideration of mental storage capacity", BEHAVIORAL AND BRAIN SCIENCES 24, 87-185.

3. Saul Sternberg (1966) "High-speed scanning in human memory", Science. 1966 Aug 5;153(736):652-4.

4. Rubin, D.C., Wenzel, A.E. (1996) "One Hundred Years of Forgetting: A Quantitative Description of Retention", Psychological Review, Vol 103, No. 4, 743-760.

5. Rubin, D.C., Hinton, S., Wenzel, A., (1999), "The Precise Time Course of Retention”, Journal of Experimental Psychology: Learning, Memory and Cognition, Vol 25, No. 5, 1161-1176.

6. Chip Quinn, personal communication.

7. Sougné, J. (1999) INFERNET: A neurocomputational model of binding and inference. doctoral dissertation, University of Liège. Collection PAIn ${ }^{\circ}$, http://www.ulg.ac.be/cogsci/jsougne/JSougneThesis.pdf, p. 23.

8. Ratcliff, R. (1978). A theory of memory retrieval. Psychological Review, 85, 59-108. 